\title{
GENERATION OF LONG WAVELENGTH HETEROGENEITY IN THE MANTLE BY THE DYNAMIC INTERACTION BETWEEN PLATES AND CONVECTION
}

\author{
Michael Gurnis and Shijie Zhong \\ Department of Geological Sciences, The University of Michigan
}

\begin{abstract}
Lateral variations in seismic velocity (through its dependence on temperature) can easily be generated at the gravest harmonics, including degrees one and two, by the dynamic interaction between plates and convection. Models of thermal convection with a single non-subducting plate have been formulated in a cylindrical geometry. Plates of width one to four times times the thickness of the convecting region strongly modulate the flow by being pushed over cold downwellings and inhibiting cooling of the fluid beneath. During rapid motion off of hot regions, a large-scale pattern of shear is developed causing small uprising limbs to be swept into the largest upwellings. Both insulation and plume-plume collisions pump energy into the lower wavenumber harmonics.
\end{abstract}

\section{Introduction}

The resolution of lateral seismic velocity variations through the deep mantle has revealed significant power at the gravest spherical harmonics, including degrees one through six [Dziewonski, et al., 1977; Masters, et al., 1982; Dziewonski, 1984; Gudmundsson, 1989; Hager and Clayton, 1989; Inoue, et al., 1990; Tanimoto, 1990]. The lower mantle body wave tomographic inversions [Dziewonski, 1984; Hager and Clayton, 1989; Inoue, et al., 1990] give comparable results at degrees two and three, but are inconsistent at shorter wavelengths [Inoue, et al., 1990]. Confidence in the long wavelength lower mantle body wave inversions is bolstered by the coherence between the observed degree two nonhydrostatic geoid and a geoid computed from dynamic flow models driven by densities constrained from tomography [Hager and Clayton, 1989; Ricard and Vigny, 1989]. Power spectra are not fully consistent at degrees one through six [Gudmundsson, 1989; Hager and Clayton, 1989; Tanimoto, 1990], but still share a fundamental attribute: a spectra with long wavelength power which is either dominated by the longest wavelengths or one which is white. In other words, for the lower mantle, power does not diminish with decreasing wavenumber. Significant heterogeneity at the gravest harmonics is a fundamental constraint on the dynamics of the

Additional graphic material is available on video tape (VHS) product code $90 \mathrm{GL} 00823 \mathrm{~V}$ : $\$ 25$. Orders must be prepaid by check, money order, or credit card. AGU accepts American Express, VISA, and MasterCard. Credit card orders may be placed through Kosmos or by calling 800-966-2481 toll free or $202-462-6900$ in D.C. or outside of the U.S. Orders placed by mail should be addressed to: AGU Caging, 2000 Florida Ave., NW, Washington, D.C. 20009; Fax: 202-328-0566; Electronic Mail: Cust. Service/Kosmos.

Copyright 1991 by the American Geophysical Union.

Paper number 91GL00823

0094-8534/91/91GL-00823\$03.00 mantle. We show that such heterogeneity can easily be generated in a system where non-subducting rafts (similar to continental lithospheric plates) are allowed to dynamically interact with thermal convection.

\section{Model Formulation}

Without any question, the model formulated here is simplified from the complexity needed to fully understand the seismic variations spectrum produced by mantle convection. The models incorporate plates, however, which have been lacking in previous studies [Jarvis and Peltier, 1990; Machetel, 1990]. The flow has been solved within a two-dimensional cylindrical geometry with gravity pointing toward the origin, $O$ (Figure 1). This geometry has important advantages over Cartesian geometry: azimuthal interconnectivity and realistic inner and outer boundaries. Azimuthal interconnectivity is vital to resolve the two-way dynamics between plates and convection [Gurnis, 1988; 1991; Gurnis and Hager, 1988]; without such interconnectivity, or periodic boundary conditions, there cannot be a mean flow between the bulk of the fluid and a plate. In addition, the scales of heterogeneity extracted with this model can be directly compared to observed scales of heterogeneity extracted with tomography.

Within the two-dimensional cylindrical geometry (Figure 1), the equations of motion, continuity, and energy are solved simultaneously for an infinite Prandtl number and incompressible fluid

$$
\begin{aligned}
& \nabla^{2} \mathrm{u}=-\nabla \mathrm{p}+\mathrm{Ra} T \hat{\hat{e}_{\mathrm{r}}} \\
& \nabla \cdot \mathrm{u}=0 \\
& \frac{\partial \mathrm{T}}{\partial \mathrm{t}}=-\mathrm{u} \cdot \nabla \mathrm{T}+\nabla^{2} \mathrm{~T}
\end{aligned}
$$

where $u=\left(u_{\theta}, u_{r}\right)$ is the velocity, $\hat{e}_{r}$ is the unit vector pointing toward the origin, $\mathrm{p}$ is the pressure, $\mathrm{T}$ is the temperature, and $\mathrm{t}$ is time. These equations have been non-dimensionalized such that the Rayleigh number and the geometry, (a-c)/a, characterize the flow. The Rayleigh number is defined as

$$
\mathrm{Ra}=\frac{p_{0} g \alpha \Delta \mathrm{TD}^{3}}{\mathrm{k} \eta}
$$

where $\alpha$ is the coefficient of thermal expansion, $\rho_{0}$ is the density, $g$ is the acceleration of gravity, $\Delta T$ is the temperature difference between the inner and outer isothermal boundaries, $\kappa$ is the thermal diffusivity, $\eta$ is the dynamic viscosity, and $D$ is the depth of the convecting layer, $a-c$, where $a$ and $c$ are the outer and inner radii, respectively. With finite elements, the equations of motion and continuity are solved with a penaltyfunction formulation and the energy equation with a Petrov- 
Galerkin method [King, et al., 1990].

A single, non-subducting plate has been incorporated into the flow using a similar technique developed for Cartesian flows [Gurnis, 1988; 1991]. The cylindrical geometry has natural periodic boundary conditions (as does a sphere) and one finite element node is pinned, forming the basis for a frame of reference. A high viscosity "rectangular" region surrounds this pinned node (lightly shaded area, Figure 1) with two weak margins on its periphery. The plate has a viscosity $10^{3}$ times the background viscosity and the weak margins are $10^{-1}$ times the background; the background is isoviscous. With respect to this pinned node, there can be a non-zero, mean velocity in the azimuthal $(\theta)$ direction. The velocity merely represents the motion of the pinned node with respect to the bulk of the fluid. The frame of reference of the system is transformed [Gurnis, 1991] by first determining the mean azimuthal velocity

$$
\omega_{\text {ref }}=\frac{1}{\pi\left(a^{2}-c^{2}\right)} \int_{S}^{u_{\theta}} d s
$$

where $S$ is the total cross-sectional area of the cylinder. Then the azimuthal offset, $\theta_{\text {ref, }}$ between the pinned node and center of mass as a function of time can be determined from

$$
\theta_{\text {ref }}=\int_{0}^{t} \omega_{\text {ref }} d t^{\prime}
$$

During post processing, visualizations of the temperature can be created by adding $-\theta_{\text {ref }}$ to the azimuthal coordinate of each node, including those defining the plate.

A number of measures have been made in order to understand the long wavelength heterogeneity and its time dependence. First, the temperature field has been deconvolved into its frequency components:

$$
\bar{T}(k)=\frac{1}{N} \sum_{n=0}^{N-1} \frac{1}{T_{2}-r_{1}} \int_{r_{1}}^{r_{2}} T(r, n \Delta \theta) d r \exp \left(-i \frac{2 \pi n}{N} k\right)
$$

where $\bar{T}(k)$ is the azimuthal Fourier transform of the temperature averaged between $r_{1}$ and $r_{2}$. In order to track the change in heterogeneity with time we have defined, $A(k)$, the relative importance of harmonic $k$ to the average of the first $L$ harmonics

$$
A(k)=L|\bar{T}(k)|\left[\sum_{j=1}^{L}|\bar{T}(j)|\right]^{-1}
$$

For all the results presented here, $\mathrm{L}=12$. A phase offset between this long wavelength heterogeneity and the position of the plate is measured by the first moment of the density distribution

$$
\begin{aligned}
& M=\int_{S} \rho x_{i} d s \hat{\hat{e}_{i}} / \int_{S} \rho d s \\
& \rho=\rho_{0}\left[1-\alpha\left(T-T_{0}\right)\right]
\end{aligned}
$$

where the components $\hat{\mathrm{e}}_{\mathrm{i}}$ are in a Cartesian system centered on the origin, 0 (Figure 1), with the $e_{2}$ direction defined by the plate. In general, $M$ depends on $\alpha$, but since we will only be interested in the orientation and relative magnitude, $\alpha$ can remain unspecified. With no boundary deformations, $M$ also represents the offset between the center of mass of the system and the geometric center. $M$ is plotted with the tail of the vector on the time axis. When the vector points toward the right, the cold area of higher density is directly underneath the plate. As shown in Figure 1, positive $\theta_{\mathrm{m}}$ is measured counterclockwise with respect to the plate.

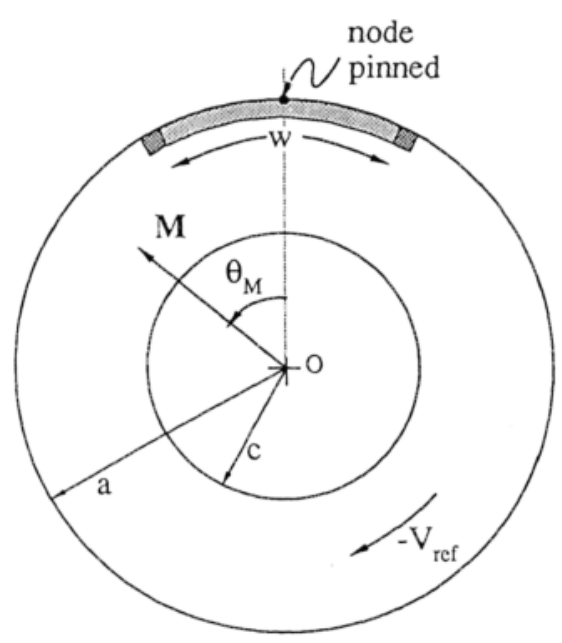

Fig. 1. Geometry of convection problem in a cylindrical geometry (see text for definition of parameters).

\section{Results}

Six cases have been solved within a mesh consisting of 33 x 241 nodes for $2.4 \times 10^{4}$ Courant time steps at $\mathrm{Ra}=1.0 \times$ $10^{5}$. The geometry was fixed such that $(a-c) / a=0.5-$ very close to the value appropriate for the whole-mantle $(0.45)$. The cases are summarized in Table 1 and animations of the entire time histories of the thermal fields and spectra are recorded on video (Appendix). The initial temperature field for all cases was $T_{0}=-\ln r / \ln 2-\varepsilon \cos \left(k_{o} \theta\right) \sin (2 \pi r)$, where $r=0.5$ at $c$ and $r=1$ at $a$. The first four cases had identical initial conditions, $\varepsilon=0.01$ and $k_{0}=2$, while the plate width, $w$, was varied between 1 and 4. Two additional cases allowed exploration of three different initial conditions, $k_{0}$, for the same plate size, $w=3$. For reference, we also ran two cases with no plates. Not surprisingly, both reached a steady-state rapidly, but with the number of cells dependent on $\mathrm{k}_{0}$. We found six cells for $k_{0}=1$ and eight for $k_{0}=4$, but in neither case was there significant power at the first or second harmonics (Table 1). However, for those cases with plates, the initial condition, $\mathbf{k}_{0}$, did not influence the time history statistics, but plate size did exert a strong influence. Because our major observation of large scale, time-dependent heterogeneity is insensitive to plate dimension, we will only concentrate on one case with a plate here.

The results for Case 3 with a plate width of three times the thickness of the convecting region is explored in detail. The time histories of the first moment of the density, $M$, mean angular velocity with respect to the plate, - $\omega_{\text {ref, }}$ and the relative amplitude of the first and second harmonics of the temperature field, $A(1)$ and $A(2)$, are shown in Figure 2. The

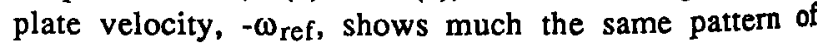


Table 1. Summary of Models

\begin{tabular}{ccccccc}
\hline Case & $w$ & $k_{0}$ & time & $\omega_{\text {ref }}{ }^{\dagger}$ & $|\overline{\mathrm{T}}(1)| \dagger$ & $|\overline{\mathrm{T}}(2)| \dagger$ \\
\hline 1 & 1 & 2 & 0.204 & 251.1 & 0.038 & 0.047 \\
2 & 2 & 2 & 0.212 & 381.2 & 0.079 & 0.040 \\
3 & 3 & 2 & 0.214 & 294.8 & 0.064 & 0.032 \\
4 & 4 & 2 & 0.216 & 274.7 & 0.082 & 0.052 \\
5 & 3 & 1 & 0.212 & 274.7 & 0.074 & 0.044 \\
6 & 3 & 4 & 0.216 & 317.6 & 0.084 & 0.039 \\
7 & 0 & 1 & 0.231 & 0.0 & $0.002^{*}$ & $0.005^{*}$ \\
8 & 0 & 4 & 0.124 & 0.0 & $0.004^{*}$ & $0.003^{*}$ \\
\hline
\end{tabular}

tPeak value.

${ }^{*}$ Steady-state value

intermittency which was discovered in a Cartesian geometry [Gumis, 1988]: periods of stationarity interrupted by shorter burst of rapid translation. The results of the other five cases are similar to the Cartesian models, except that for smaller plates periods of stationarity are much longer and well defined and interrupted by short bursts of plate velocity (see video). For larger plate widths, $w=4$, the plate is almost always in motion, although still time-dependent (see video). The intermittency is due primarily to the insulation of the interior of the fluid by the relatively thick plate [Gumis, 1988]. The plate rotates with respect to the first moment of the density, $M$, and the intermittency of plate velocity is in phase with fluctuations in the strength of the first and second harmonics of the temperature, $A(1)$ and $A(2)$. During peak plate velocity $M$ is perpendicular to the plate center and $A(1)$ and $A(2)$ reach their maximum. The controlling physics is quite evident in the animations, but can also be understood in the context of the four thermal fields shown in Figure 3. The spectra, $|\overline{\mathrm{T}}(\mathrm{k})|$ versus $k$, at these four instants are shown in Figure 4.

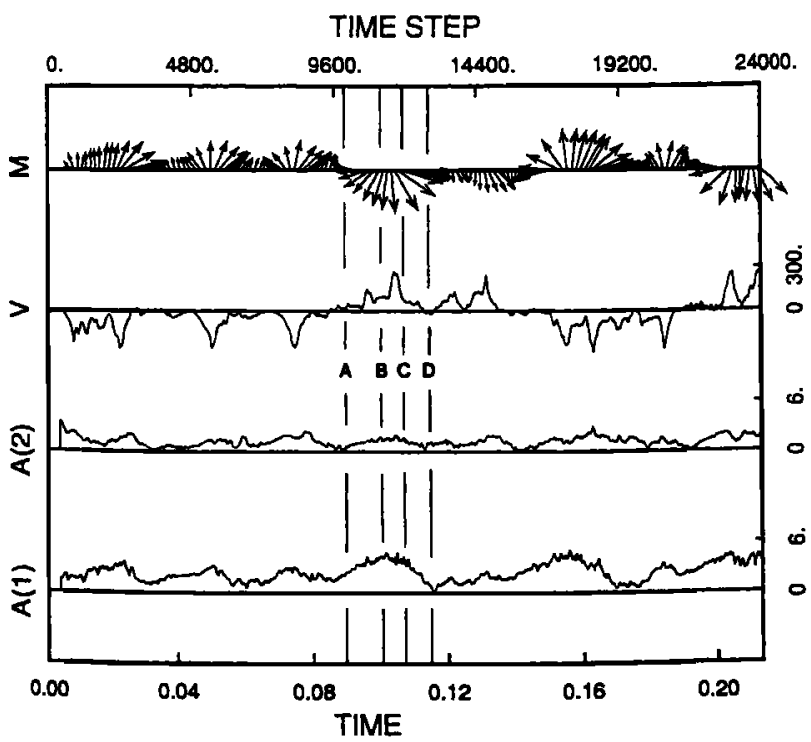

Fig. 2. Time history of first moment of the temperature field, $M$, mean angular velocity of the plate, $\omega_{\text {ref }}$ (labelled $V$ in the figure), and relative strengths of the first and second harmonics of the temperature field, $A(1)$ and $A(2)$ for Case 3. The vertical lines labelled A-D denote times for which the temperature fields and spectra are shown in Figures 3 and 4.
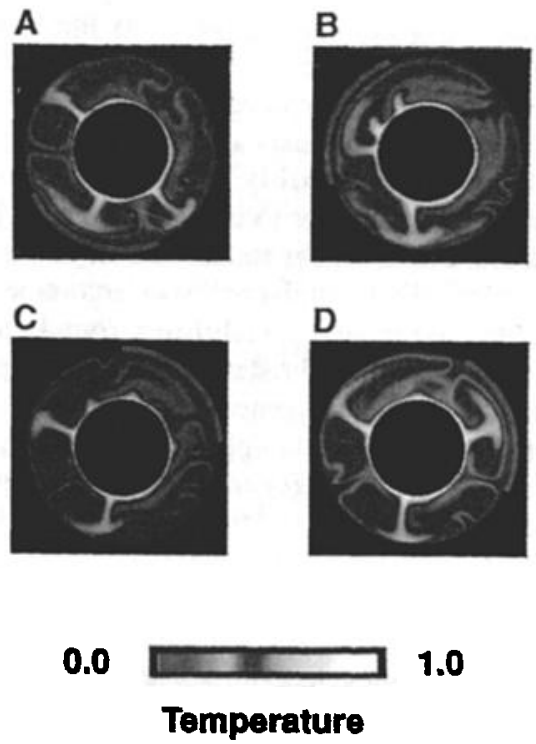

Fig. 3. Temperature fields and plate locations for the times indicated in Figure 2. The locations of the plate is denoted by the green "rectangle" on the top of the outer boundary. The images are in the "mantle" reference frame. The times of the frames are 0.091 (A), 0.102 (B), 0.108 (C), and 0.115 (D).

At time $=0.091$ (Figure 3a) the plate is relatively stationary and $M$ points $\pi$ radians away from the plate; although $A(2)$ is at an approximate minimum, $A(1)$ is growing out of a minimum as the temperature under the plate increases. The spectra is skewed toward smaller harmonics (Figure 4a). The plate soon starts to move in a positive direction and $M$ rotates through $\pi / 2$ (Figure $3 \mathrm{~b}$ ) as the first harmonic continues to growth (Figure 2); the low order spectra is dominated by the first harmonic (Figure $4 \mathrm{~b}$ ). $M$ rotates to 0 , as the plate settles in over the "cold" hemisphere which gives rise to so much power at $\mathrm{k}=1$ (Figure $3 c$ ). $|\overline{\mathrm{T}}(1)|$ and $|\overline{\mathrm{T}}(2)|$ diminish in strength and new hot upwellings are established under the plate which has again become stationary by time 0.115 (Figure $3 \mathrm{~d}$ and $4 \mathrm{~d}$ ). During lateral translation of the plate, plumeplume collisions maintain the strength of the large-scale flow

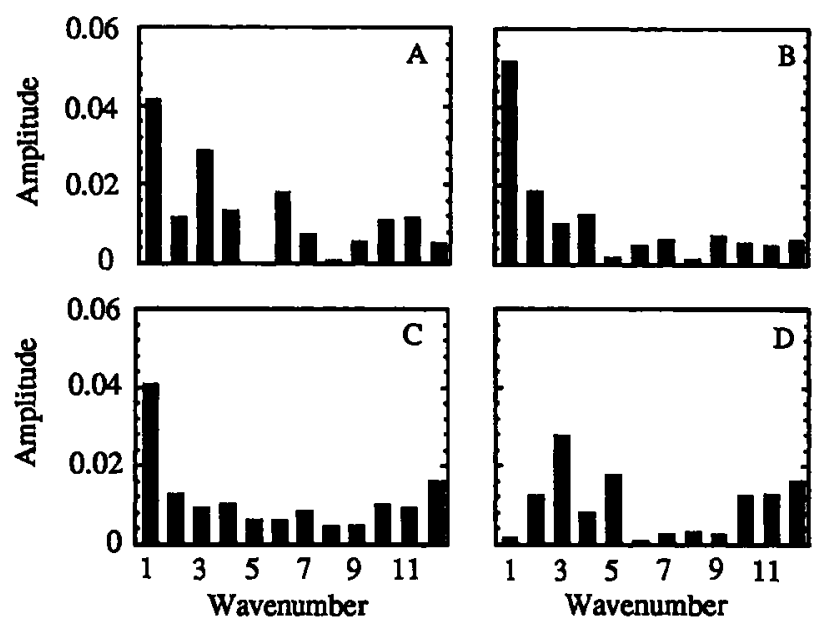

Fig. 4. Spectra of the average temperature field between $r_{1}=$ 0.625 and $r_{2}=0.875$ for the first 12 harmonics. 
(video). This is evident in Figure $3 \mathrm{~b}$ as the three uprising limbs under the plate merge into one. When the flow is highly unsteady and the flow dominated by a large-scale circulation, small plumes are continuously swept into the larger plumes. Indeed, the plumes probably move towards a common centroid, a thermal attractor [Vincent and Yuen, 1988], such that the largest limbs appear to be relatively stationary. This large scale circulation is similar to stable large-aspect ratio cells with traveling boundary instabilities found in Cartesian geometry with no plates [Christensen, 1987; Hansen and Ebel, 1988]. Plate insulation may generate the large scale circulation (and eventually destroy it), but plume-plume collisions tend to reinforce and maintain longer wavelength components of the temperature field.

\section{Conclusions}

Current tomography techniques smooth and distort the spectra of mantle anomalies, but this does not mean we should rule out the possibility the Earth has gross structure. Indeed, the ability to predict the degree two and three geoid with seismic tomography [Hager and Clayton, 1989; Ricard and Vigny, 1989] strongly suggests that the Earth has gross structure and that it is partly resolved with seismic tomography. Past studies have shown that isoviscous convection with stress free boundary conditions is incapable of producing significant power at low wavenumbers [Jarvis and Peltier, 1990; Machetel; 1990]. We have shown here that once a non-subducting plate is introduced, however, heat loss is laterally inhibited and large-scale temperature variations (internal heterogeneity) become a dominant feature of the flow.

\section{Appendix: Video}

Animations of the temperature fields and spectra for the first six cases with plates have been recorded on video. The frames which make up the video are each separated by 20 computational time steps. The first 400 time steps are not presented. The spectral amplitudes are normalized by the maximum amplitude which occurred after the initial transient overturn. The videos are shown as a repeating loop and two complete cycles are shown; the window containing the thermal field and spectra blanks just before the cycle repeats.

Acknowledgments. Funded by NSF grants EAR-8957164 and EAR-8904660.

\section{References}

Christensen, U. R., Time-dependent convection in elongated Rayleigh-Benard cells, Geophys, Res. Lett. 14, 220-223, 1987.

Dziewonski, A. M., Mapping the lower mantle: Determination of lateral heterogeneity in $P$ velocity up to degree and order 6, J. Geophys. Res., 89, 5929-5952, 1984.

Dziewonski, A. M., Hager, B. H., and O'Connell, R. J.,
Large-scale heterogeneities in the lower mantle, $\underline{I}$. Geophys. Res, 82, 239-255, 1977.

Gudmundsson, O., Some Problems in Global Tomography, Ph. D. Thesis, California Institute of Technology, Pasadena, CA, 225 p., 1989.

Gurnis, M., Large-scale mantle convection and the aggregation and dispersal of supercontinents, Nature, 332. 695-699, 1988.

Gurnis, M., Continental flooding and mantle-lithosphere dynamics, in R. Sabadini and K. Lambeck (eds), Glacial Isostasy. Sea-Level and Mantle Rheology, Kluwer Academic Publishers, Dordrect, in press, 1991.

Gurnis, M. and Hager, B. H., Controls on the structure of subducted slabs, Nature, 335, 317-322, 1988.

Hager, B. H. and Clayton, R. W., Constraints on the structure of mantle convection using seismic observations, flow models, and the geoid, in R. W. Peltier (ed.) Mantle Convection, Gordon and Breach Science Publishers, New York, pp. 657-763

Hansen, U. and Ebel, A., Time-dependent thermal convection -- a possible explanation for a multiscale flow in the Earth's mantle, Geophys. J. 94, 181-191, 1989.

Inoue, H., Fukao, Y., Tanabe, K., and Ogata, Y., Whole mantle P-wave travel time tomography, Phys. Earth Planet. Inter.59, 294-328, 1990.

Jarvis, G. T. and Peltier, W. R., Low-wavenumber signatures of time-dependent mantle convection, Phys. Earth Planet.Inter. 59, 182-194, 1990.

King, S. D., A. Raefsky, and B. H. Hager, ConMan: Vectorizing a finite element code for incompressible twodimensional convection in the Earth's mantle, Phys. Earth Planet. Inter. 59, 195-207, 1990.

Machetel, P., Short-wavelength lower mantle seismic velocity anomalies, Geophys. Res. Lett. 17, 1145-1148, 1990.

Masters, G., Jordon, T. H., Silver, P. G., and Gilbert, F., Aspherical Earth structure from fundamental spheroidalmode data, Nature. 298, 609-613, 1982.

Ricard, Y. and Vigny, C., Mantle dynamics with induced plate tectonics, J. Geophys. Res. 94, 17,543-17,559, 1989.

Tanimoto, T., Long-wavelength S-wave velocity structure throughout the mantle, Geophys. J. Int. 100, 327-336, 1990.

Vincent, A. P. and Yuen, D. A., Thermal attractor in chaotic convection with high-Prandtl-number fluids, Phys. Rev. A. 38, 328-334, 1988.

M. Gurnis and S. Zhong, Dept. of Geological Sciences, Univ. of Michigan, Ann Arbor, 48109-1063.

(Received: September 21, 1990; revised: January 14, 1991; accepted: January 17, 1991.) 